CLINICAL STUDY

\title{
Diabetes reduces left ventricular ejection fraction-irrespective of presence and extent of coronary artery disease
}

\author{
Niklas F Ehl ${ }^{1}$, Michael Kühne ${ }^{1}$, Miriam Brinkert ${ }^{1}$, Jan Müller-Brand ${ }^{2}$ and Michael J Zellweger ${ }^{1}$ \\ Departments of ${ }^{1}$ Cardiology and ${ }^{2}$ Nuclear Medicine, University Hospital, Petersgraben 4, CH - 4031 Basel, Switzerland \\ (Correspondence should be addressed to M J Zellweger; Email: mzellweger@uhbs.ch)
}

\begin{abstract}
Background: It is not clear whether diabetes reduces systolic left ventricular function (left ventricular ejection fraction, LVEF) irrespective of coronary artery disease (CAD). The aim of this study was to compare the LVEF between diabetic and non-diabetic patients with respect to the extent of CAD.

Methods and results: Consecutive patients undergoing stress myocardial perfusion SPECT (MPS) were evaluated. MPS was interpreted using a 20-segment model with a five-point scale to define summed stress score (SSS), summed rest score, and summed difference score. LVEF was measured by gated SPECT and then compared with respect to diabetic status and SSS categories. Of 2635 patients, data of 2400 was available. Of these, $24 \%$ were diabetic, mean age was $64 \pm 11 \mathrm{y}$, and $31 \%$ were female. Diabetics had a significantly lower LVEF compared with non-diabetics regardless of the extent of CAD: $53 \pm 13$ and $55 \pm 13 \%$ respectively $(P=0.001)$. Diabetics and non-diabetics did not differ significantly in the distribution of SSS categories. Diabetes was an independent predictor of decreased LVEF (odds ratio $1.6,95 \%$ confidence interval $1.2-2.0 ; P<0.001$ ).

Conclusion: Diabetics had a lower LVEF than non-diabetics. This difference could be demonstrated regardless of CAD extent and might in part explain their generally worse cardiac survival compared with non-diabetics on an epidemiological level. In addition, this finding points to discussed mechanisms other than CAD lowering LVEF in diabetics.
\end{abstract}

European Journal of Endocrinology 165 945-951

\section{Introduction}

The association between diabetes and congestive heart failure (CHF), as well as its influence on prognosis, is well known and has been extensively documented (1-4); it was established in the study by Framingham in 1971 in which the high prevalence of diabetes ( $14 \%$ of men and $26 \%$ of women) among patients with CHF was noted $(5,6)$. However, generally this finding was explained by increased prevalence of coronary artery disease (CAD) in diabetic patients $(7,8)$. However, large epidemiological studies have confirmed diabetes mellitus to be an independent risk factor for the development of heart failure (9); Rubler et al. (10) first described the existence of 'diabetic cardiomyopathy' in 1972 based on four adult diabetic patients with CHF that could not be explained by CAD, hypertension, valvular heart disease, or alcoholism. Subsequently, the term 'diabetic cardiomyopathy' as a diastolic and/or systolic CHF in diabetic patients in the absence of significant concomitant $\mathrm{CAD}$ or arterial hypertension has been defined.

In contrast to the consensus that diastolic dysfunction plays an important role regarding CHF in diabetic patients, the results regarding the systolic function were not conclusive. Taking also into account the presence and extent of CAD, little is known about the comparison of left ventricular ejection fraction (LVEF) in diabetic vs non-diabetic patients. It is well known that LVEF is an important prognostic variable (11). Therefore, the aim of our study was to compare the LVEF of diabetic and non-diabetic patients adjusted for the presence and extent of CAD.

\section{Subjects and methods}

\section{Study population}

A large cohort of 2635 consecutive patients who underwent exercise or adenosine stress myocardial perfusion SPECT (MPS) at our institution was retrospectively evaluated. Patients with clinically relevant valvular disease were excluded from the study population. Of these 2635 patients, two were excluded for unknown diabetic status and 233 were excluded for technical reasons (LVEF could not be assessed by gated SPECT, mostly due to supraventricular or ventricular premature beats or atrial fibrillation). The final patient population consisted of 2400 patients: 1826 non-diabetic and 574 diabetic patients. 
Diagnosis of diabetes mellitus was based on a known history of diabetes and/or antidiabetic drug therapy at the time of stress testing.

A detailed patient interview and chart review of former cardiac evaluations were the basis to assess the medical history of the patients.

\section{Rest/stress MPS protocol}

MPS was performed using a single-day gated rest/stress dual isotope protocol (TI-201 rest/Tc-99m sestamibi stress as described previously $(12,13))$. Whenever possible, $\beta$-blockers as well as negative chronotropic calcium antagonists were withheld for $48 \mathrm{~h}$ and longacting nitrates for $24 \mathrm{~h}$ before exercise testing; patients were instructed to avoid caffeine-containing products for $24 \mathrm{~h}$ prior to MPS. Ten minutes after administration of 111 MBq Thallium 201, a rest SPECT was obtained. Whenever possible, a symptom-limited exercise treadmill test using standard protocols was performed with a 12-lead electrocardiogram (ECG) recorded each minute. At near-maximal stress, a $740 \mathrm{MBq}$ dose of Tc-99m sestamibi was injected, and exercise continued for at least an additional minute after injection.

If exercise stress was not possible or sufficient, a pharmacologic one (adenosine $140 \mu \mathrm{g} / \mathrm{kg}$ per min for 6 min) was used either combined with physical stress or alone. Tc-99m sestamibi was injected after $3 \mathrm{~min}$ of adenosine infusion.

During both types of stress, blood pressure and ECG were monitored according to the standard protocols $(13,14)$.

\section{Image acquisition}

SPECT was performed using triple-head gamma cameras (IRIX3, PRISM3000, PHILIPS, CH) equipped with a low-energy ultra high-resolution collimator. A 40 and $20 \%$ symmetrical energy window was used to acquire the 72 and $169 \mathrm{keV}$ peak of thallium, respectively, and a $15 \%$ symmetrical energy window to acquire the $140 \mathrm{keV}$ peak of technetium. One hundred and twenty images $(64 \times 64$ matrix $)$ were acquired with 15 s per projection for stress and 20s for rest images. Poststress images were gated with eight frames per heartbeat. Transaxial, sagittal, and coronal tomograms were reconstructed using a low-pass filter during back projection. Imaging was performed using standard protocols $(12,15)$. No attenuation or scatter correction was used. Instead supine and prone images were taken.

\section{Analysis of perfusion SPECT}

Semiquantitative visual interpretation was performed using a 20-segment model (each segment representing $5 \%$ of the myocardium), as described previously $(13,16)$. MPS was read by two experienced readers, a cardiology and a nuclear medicine physician. Radioisotope uptake in each segment was graded using a five-point scale $(0=$ normal, $1=$ equivocal, $2=$ moderate, $3=$ severe reduction of radioisotope uptake, and $4=$ apparent absence of detectable tracer uptake in a segment). A summed stress score (SSS) was obtained by adding the scores of the 20 segments on the stress images, a summed rest score (SRS, reflecting in part the extent of scar and thus prior myocardial infarction) in a similar way by adding the scores of the 20 segments on the rest images. To assess the extent and severity of ischemia, a summed difference score (SDS, as degree of defect reversibility and extent of ischemia) was calculated by subtracting SRS from SSS. A SSS $<4$ was considered as a normal, SSS 4-8 mildly abnormal, SSS 9-13 moderately abnormal, and SSS $>13$ severely abnormal perfusion (17). A transient ischemic dilatation (TID) ratio of more than 1.22 was considered positive. TID is a marker of potentially balanced and/or potentially severe ischemia.

\section{LVEF evaluation}

LVEF was calculated by using the widely validated Quantitative Gated SPECT (QGS, Cedars Sinai, Inc., Los Angeles, CA, USA) software (18). By this algorithm, the left ventricle is segmented into short axis images with eight frames per cardiac cycle. Endocardial and epicardial surfaces and valve plane for every gating interval were determined. LVEF was derived from calculated LVEDV and LVESV. LVEF assessment by MPS is well validated and reproducible $(19,20)$.

LVEF $\geq 50 \%$ was considered as normal and $<50 \%$ as reduced (21).

\section{Statistical analysis}

Continuous variables are described as the mean \pm s.D. for the equally distributed and as median for the nonequally distributed variables.

Categorical and ordinal variables were compared using a $\chi^{2}$ test. Continuous variables were compared using the T-test, Mann-Whitney $U$ and one-way ANOVA with Bonferroni correction as appropriate.

A multivariate linear regression model was built to evaluate the influence of all significantly different variables of Tables 1 and 2 on LVEF. In addition to diabetes, SRS (as a surrogate marker of prior myocardial infarction/scar) and SDS (as a marker of ischemia) were part of the model to correct for the presence and extent of CAD.

A multivariate analysis (bivariate logistic regression) was performed to evaluate the independent predictors of decreased LVEF. The same variables as in the multiple linear regression model were incorporated into the logistic regression model. $P$ value, odds ratio, and confidence interval (CI) were calculated for each variable. 
Table 1 Baseline characteristics.

\begin{tabular}{lllll}
\hline & $\begin{array}{l}\text { Overall } \\
(n=2400)\end{array}$ & $\begin{array}{l}\text { Non-diabetics } \\
(n=1826)\end{array}$ & $\begin{array}{l}\text { Diabetics } \\
(n=574)\end{array}$ & $\boldsymbol{P}$ \\
\hline Mean age \pm s.D. & $64 \pm 11$ & $64 \pm 11$ & $65 \pm 10$ & $\mathbf{0 . 0 1 6}$ \\
Female & $736(30.7 \%)$ & $579(31.7 \%)$ & $157(27.4 \%)$ & $\mathbf{0 . 0 4 9}$ \\
BMI $\left(\mathrm{kg} / \mathrm{m}^{2}\right) \pm$ s.D. & $27.5 \pm 4.7$ & $27.0 \pm 4.4$ & $29.2 \pm 5.0$ & $<\mathbf{0 . 0 0 1}$ \\
Typical angina & $802(33.4 \%)$ & $643(35.2 \%)$ & $159(27.7 \%)$ & $\mathbf{0 . 0 0 5}$ \\
Atypical angina & $648(27.0 \%)$ & $514(28.1 \%)$ & $134(23.4 \%)$ & 0.058 \\
Dyspnea & $955(39.8 \%)$ & $705(38.6 \%)$ & $250(43.6 \%)$ & 0.064 \\
Known CAD & $1106(46.1 \%)$ & $857(46.9 \%)$ & $249(43.4 \%)$ & 0.412 \\
Prior Ml & $776(32.3 \%)$ & $662(33.2 \%)$ & $162(28.2 \%)$ & 0.099 \\
Dyslipidemia & $1393(58.1 \%)$ & $1012(55.5 \%)$ & $381(66.5 \%)$ & $<\mathbf{0 . 0 0 1}$ \\
Arterial hypertension & $1556(64.9 \%)$ & $1103(60.4 \%)$ & $453(78.9 \%)$ & $<\mathbf{0 . 0 0 1}$ \\
Family history of CAD & $649(27.1 \%)$ & $506(27.8 \%)$ & $143(25.0 \%)$ & 0.200 \\
Smoker & $1104(46.0 \%)$ & $858(47.0 \%)$ & $246(42.9 \%)$ & $\mathbf{0 . 0 4 0}$ \\
Aspirin & $1900(72.1 \%)$ & $1326(72.6 \%)$ & $411(71.6 \%)$ & 0.631 \\
Anticoagulation & $212(8.8 \%)$ & $153(8.4 \%)$ & $59(10.3 \%)$ & 0.177 \\
$\beta-B l o c k e r s$ & $1512(63.0 \%)$ & $1172(64.2 \%)$ & $340(59.3 \%)$ & $\mathbf{0 . 0 4 0}$ \\
ACE inhibitors & $487(28.9 \%)$ & $270(21.5 \%)$ & $169(38.9 \%)$ & $<\mathbf{0 . 0 0 1}$ \\
ATII antagonists & $401(23.8 \%)$ & $289(21.5 \%)$ & $131(30.1 \%)$ & $<\mathbf{0 . 0 0 1}$ \\
Calcium antagonists & $478(20.0 \%)$ & $334(18.3 \%)$ & $144(25.2 \%)$ & $<\mathbf{0 . 0 0 1}$ \\
Nitrates & $312(13.1 \%)$ & $238(13.1 \%)$ & $74(13.0 \%)$ & 1 \\
Statins & $1208(53.6 \%)$ & $889(52.3 \%)$ & $319(57.6 \%)$ & $\mathbf{0 . 0 3 0}$
\end{tabular}

BMI, body mass index; MI, myocardial infarction; CAD, coronary artery disease. Statistically significant values are in boldface.

A $P$ value $<0.05$ was considered statistically significant.

The analyses were performed with SPSS version 19.0 (SPSS, Inc., Chicago, IL, USA).

\section{Results}

\section{Baseline data}

Table 1 summarizes the baseline characteristics of the whole patient population and the comparison of diabetic and non-diabetic patients.

Diabetic patients were older, less often female, had a higher body mass index (BMI), more often had a history of dyslipidemia and arterial hypertension, but less often were smokers. They were more often treated with statins, ACE inhibitors, ATII- and calcium antagonists, but less often with $\beta$-blockers, compared with their non-diabetic counterparts. Of note, diabetic patients reported typical angina less frequently than non-diabetic patients. Of the 574 diabetic patients, 120 were treated with insulin, 314 with an oral antidiabetic therapy, and for the remaining 140 patients the treatment modality was not known.

\section{Stress test and MPS results}

The MPS results of diabetic and non-diabetic patients are shown in Table 2.

Non-diabetic patients had a higher resting heart rate, were more often able to undergo physical stress and complained more often of typical anginal chest pain during stress testing. Regarding overall MPS abnormality (SSS) and ischemia (SDS), there was no difference

Table 2 Results stress/myocardial perfusion SPECT.

\begin{tabular}{llllr}
\hline & $\begin{array}{l}\text { Overall } \\
(n=2400)\end{array}$ & $\begin{array}{l}\text { Non-diabetics } \\
(n=1826)\end{array}$ & $\begin{array}{l}\text { Diabetics } \\
(n=574)\end{array}$ & $\boldsymbol{P}$ \\
\hline Resting heart rate $(\mathrm{bpm})$ & $78 \pm 16$ & $76 \pm 16$ & $81 \pm 16$ & $<\mathbf{0 . 0 0 1}$ \\
Sinus rhythm & $2300(95.8 \%)$ & $1753(96.0 \%)$ & $547(95.3 \%)$ & 0.290 \\
LBBB & $95(4.0 \%)$ & $68(3.7 \%)$ & $27(4.7 \%)$ & 0.440 \\
Physical stress & $1528(63.7 \%)$ & $1217(66.6 \%)$ & $311(54.2 \%)$ & $<\mathbf{0 . 0 0 1}$ \\
Angina during stress & $592(24.7 \%)$ & $469(25.7 \%)$ & $123(21.4 \%)$ & $\mathbf{0 . 0 4 1}$ \\
ST changes & $459(19.1 \%)$ & $360(19.7 \%)$ & $99(17.3 \%)$ & 0.181 \\
Antianginal therapy stopped & $1037(43.3 \%)$ & $787(43.1 \%)$ & $250(43.6 \%)$ & 0.855 \\
SSS (median) & 5 & 5 & 6 & 0.829 \\
SRS (median) & 1 & 1 & 2 & 0.401 \\
SDS (median) & 2 & 2 & 2 & 0.710 \\
LVEF \pm s.D. & $54 \pm 13$ & $55 \pm 13$ & $53 \pm 13$ & $\mathbf{0 . 0 0 1}$ \\
TID & $186(7.8 \%)$ & $125(6.9 \%)$ & $61(10.7 \%)$ & $\mathbf{0 . 0 0 5}$ \\
\hline
\end{tabular}

bpm, beats per minute; LBBB, left bundle branch block; SSS, summed stress score; SRS, summed rest score; SDS, summed difference score; TID, transient ischemic dilatation. Statistically significant values are in boldface. 
Table 3 Distribution in four SSS subgroups.

\begin{tabular}{lccc}
\hline & $\begin{array}{c}\text { Non-diabetics } \\
(n=1826)(\%)\end{array}$ & $\begin{array}{c}\text { Diabetics } \\
(n=574)(\%)\end{array}$ & $\boldsymbol{P}$ \\
\hline SSS $<4$ & 41 & 39 & 0.21 \\
SSS 4-8 & 22 & 23 & 0.76 \\
SSS 9-13 & 15 & 18 & 0.23 \\
SSS $>13$ & 22 & 20 & 0.62
\end{tabular}

SSS, summed stress score.

between the groups; however, TID was less often observed in non-diabetic patients.

Overall LVEF was significantly lower in diabetic compared with non-diabetic patients, $55 \pm 13$ and $53 \pm 13 \%$ respectively $(P=0.001)$.

\section{Test results with respect to CAD extent}

All patients were stratified into four subgroups with respect to CAD extent, as defined by the SSS. Distribution of diabetic and non-diabetic patients in these subgroups was similar which is shown in Table 3. The median SSS and SDS were also similar in these four subgroups ( $P$ not significant for all comparisons).

Diabetic patients had a significantly lower LVEF than non-diabetic patients irrespective of CAD extent (Fig. 1).

\section{Relation to antidiabetic treatment}

In a subgroup analysis, we evaluated the LVEF regarding the antidiabetic treatment (Fig. 2). The LVEF turned out to be significantly lower in patients treated with insulin in comparison with non-diabetic patients and diabetic patients without insulin treatment. No difference could be shown between non-diabetic patients and diabetic patients that were not treated with insulin.

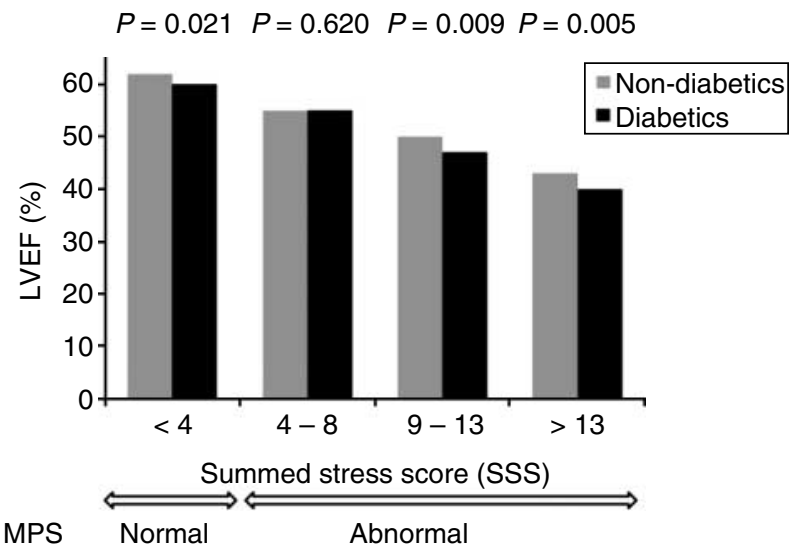

Figure 1 Comparison of LVEF in non-diabetic and diabetic patients regarding as a function of coronary artery disease $(C A D)$ - in three of the four subgroups, a significantly higher LVEF in non-diabetic compared with diabetic patients could be shown irrespective of CAD extent. LVEF, left ventricular ejection fraction; SSS, summed stress score; MPS, myocardial perfusion SPECT.

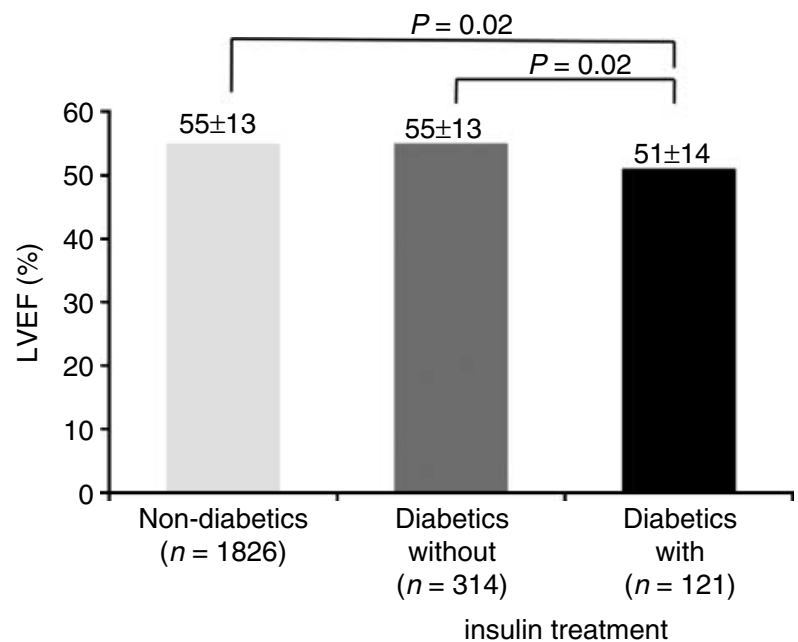

Figure 2 LVEF difference regarding the antidiabetic treatment regimen in the diabetic patients - significant differences were only seen in the subgroup of diabetic patients treated with insulin: their LVEF was significantly lower in comparison with non-diabetic patients and diabetic patients without insulin treatment. LVEF, left ventricular ejection fraction.

\section{Variables linked to LVEF}

In the multivariate linear regression analysis, male sex, diabetes, ACE inhibitor intake, pharmacologic stress, SRS, and SDS were linked with decrease in LVEF. Typical angina and intake of a statin were linked with increase in LVEF (Table 4).

\section{Independent predictors of decreased LVEF}

In total, 545 non-diabetic patients (30\%) and 211 diabetic patients $(37 \%)$ had a reduced LVEF $(P=0.005)$.

As shown in Table 5, male sex, diabetes, pharmacological stress, SRS, and SDS were independent predictors of reduced LVEF. In contrast, the presence of angina decreased the probability of reduced LVEF. Of note, TID (as a possible marker for balanced and/or extensive ischemia) was not a predictor of reduced LVEF.

Diabetes, therefore, independently predicted decreased LVEF irrespective of prior CAD, evidence and extent of CAD.

\section{Discussion}

This study demonstrated a significantly lower LVEF in diabetic compared with non-diabetic patients $(P=0.001)$ in a large patient population. Even though the difference was small, this finding may have important epidemiological impact, since LVEF is one of the most important predictors of survival. This hypothesis is supported by data from the Mayo clinic that has demonstrated a significantly worse survival of even asymptomatic diabetic patients with an LVEF of $<50 \%$ (22). 
Table 4 Variables independently influencing LVEF (as assessed by linear regression analysis).

\begin{tabular}{lrcr}
\hline & $\begin{array}{c}\text { Regression } \\
\text { coefficient }\end{array}$ & 95\% Cl & $\boldsymbol{P}$ \\
\hline Male sex & -6.72 & -7.72 to -5.72 & $<0.001$ \\
Typical angina & 1.73 & 0.61 to 2.85 & 0.002 \\
Diabetes & -1.62 & -2.67 to -0.57 & 0.003 \\
Intake of ACE inhibitor & -1.75 & -2.79 to -0.71 & 0.001 \\
Intake of a statin & 1.35 & 0.42 to 2.28 & 0.005 \\
Pharmacological stress & -2.27 & -3.21 to -1.32 & $<0.001$ \\
SRS (/score point) & -1.03 & -1.11 to -0.95 & $<0.001$ \\
SDS (/score point) & -0.46 & -0.56 to -0.36 & $<0.001$ \\
\hline
\end{tabular}

SRS, summed rest score; SDS, summed difference score.

The major finding of this study was that the difference in LVEF held true irrespective of the presence and extent of CAD.

In the last decades, the systolic function in diabetic vs non-diabetic patients has been investigated repeatedly, although mostly in cohorts with much fewer patients and with different LVEF measurement methods; in addition, the results were not conclusive: regarding LVEF, most of the studies did not report a difference between diabetic and non-diabetic patients (23-25), despite some studies that demonstrated a slightly lower LVEF in diabetic patients $(26,27)$. In the studies in which a lower LVEF was shown, it was mainly attributed to the fact that CAD is more common and also more often severe in diabetic patients even though the presence and extent of CAD were not taken into account $(22,28-30)$. This gap has been closed with our study because LVEF was compared in patients without evidence of $\mathrm{CAD}$ or in comparable groups when it came to the extent of CAD.

Konduracka et al. (24) also did not find any difference in LVEF $(P=0.169)$ in a relatively small patient cohort (185 diabetic patients and 105 non-diabetic control subjects) and raised the question whether diabetes alone was really able to precipitate myocardial dysfunction despite the well-known histological changes in the myocardium; however, they only assessed patients with type 1 diabetes without risk factors and a mean age of only 34.5 years.

Only a few smaller studies have taken the presence of CAD into account, e.g. Htay et al. (23) evaluated the LVEF in 70 patients with diabetes mellitus type 2 and compared the results with gender-matched controls; after having excluded the presence of CAD, mean LVEF was shown to be comparable at least in men with and without diabetes $(P=0.16)$ but was lower in women with diabetes compared with women without diabetes $(P=0.04)$.

LVEF of patients treated with insulin was significantly lower in comparison with non-diabetic patients $(P=0.02)$ and diabetic patients $(P=0.02)$ without insulin treatment (even though insulin treatment did not turn out as an independent predictor of decreased LVEF). These results are in line with other observations where insulin treatment or insulin resistance was predictive of CHF development $(4,31)$. In addition, Smooke et al. (32) showed an independently and markedly increased risk of mortality in insulin-treated diabetic patients with CHF (hazard ratio (HR) 4.30, 95\% CI 1.69-10.94, $P<0.00001)$, but not in non-insulintreated diabetic patients compared with non-diabetic patients (HR 0.95, 95\% CI 0.31-2.93, $P=0.395$ ). Witteles \& Fowler (33) pointed out that there is increasing evidence that insulin resistance is also a primary etiologic factor in the development of non-ischemic CHF; indeed, insulin has profound effects on the myocardium (regarding energy metabolism, sympathetic activity, inflammation, oxidative stress, and microvascular dysfunction) $(24,34)$, but insulin treatment also implicates greater extent of macrovascular disease (a fact that did not seem to play a role in our study).

In this study, LVEF decreased as a function of diabetes in addition to male sex, extent of CAD (scar as shown by SRS; ischemia as represented by SDS), pharmacologic stress, and ACE inhibitor intake. Male individuals are well known to have lower LVEF than female individuals (35). The correlation of the extent of CAD and LVEF is quite obvious and pharmacologic stress is a well-known surrogate marker for overall sicker patients. ACE inhibitor intake is of course more common in patients with reduced LVEF. Interestingly, the presence of typical angina was linked to a higher LVEF. This is in line with the seemingly paradoxical finding in a large patient population where angina turned out to be a 'protective' factor (36). This may be similar to the fact that symptomatic patients seek help before they have an event as in contrast to patients with silent events, e.g. a silent myocardial infarction that results in decreased LVEF. A statin therapy demonstrated a positive correlation with LVEF. A recent meta-analysis also showed a positive effect of statin treatment on LVEF by demonstrating that use of statins in CHF patients may result in the improvement of cardiac function (37).

Looking at variables independently predicting a decreased LVEF, diabetes turned out to be an independent predictor, this in addition to male sex, pharmacologic stress, extent of scar (SRS), and ischemia (SDS). Again, patients with typical angina were less likely to have a decreased LVEF. Potential reasons have been discussed above.

Table 5 Independent predictors of decreased LVEF.

\begin{tabular}{lccr}
\hline & OR & $\mathbf{9 5 \% ~ C l}$ & \multicolumn{1}{c}{$\boldsymbol{P}$} \\
\hline Male sex & 2.0 & $1.40-2.81$ & $<0.001$ \\
Typical angina & 0.7 & $0.47-0.96$ & 0.026 \\
Diabetes & 1.6 & $1.14-2.13$ & 0.006 \\
Pharmacologic stress & 1.7 & $1.25-2.23$ & 0.001 \\
SRS (/score point) & 1.32 & $1.28-1.37$ & $<0.001$ \\
SDS (/score point) & 1.11 & $1.07-1.14$ & $<0.001$
\end{tabular}

SRS, summed rest score; SDS, summed difference score. 


\section{Limitations}

We acknowledge the potential limitations of our study. It was a retrospective evaluation; although we recorded antidiabetic management at baseline, information on the duration of diabetes or diabetic control (such as $\mathrm{HbA1c)}$ was not available. As our patients were consecutive referrals to a tertiary center for further evaluation, there is certainly a referral bias.

Furthermore, we used MPS variables as surrogate markers for the presence and extent of CAD. Of course, by this method, we only pick up changes that are hemodynamically relevant and do not provide information on the coronary artery level (e.g. calcification). However, for the purpose of our study, this point should not play a relevant role.

The treatment modality in diabetic patients could not be assessed in 140/574 patients. In addition, we could not determine the type of diabetes (although we estimate more than $95 \%$ to be of type 2 ).

\section{Implications}

The high prevalence, morbidity, and mortality of CHF mandate early identification of risk factors and clinical signs to start appropriate and timely therapy; in addition to the high prevalence of CHF in diabetic patients, diabetes has been shown to have a prognostic impact on these patients $(38,39)$. Detecting and understanding LV dysfunction in diabetic patients may also help to delay or prevent the progression of CHF.

\section{Conclusion}

Diabetic patients had a lower LVEF than non-diabetic patients (even though the overall difference was small). This difference could be demonstrated regardless of $\mathrm{CAD}$ extent and may in part explain their worse cardiac survival compared with non-diabetic patients on an epidemiological level. In addition, this finding points to other discussed mechanisms than CAD being responsible for decreased LVEF in diabetic patients.

\section{Declaration of interest}

The authors declare that there is no conflict of interest that could be perceived as prejudicing the impartiality of the research reported.

\section{Funding}

This research did not receive any specific grant from any funding agency in the public, commercial, or not-for-profit sector.

\section{Acknowledgements}

This work has been presented in part at the annual meeting of the European Society of Cardiology in Munich, 2008.

\section{References}

1 MacDonald MR, Petrie MC, Hawkins NM, Petrie JR, Fisher M, McKelvie R, Aguilar D, Krum H \& McMurray JJ. Diabetes, left ventricular systolic dysfunction, and chronic heart failure. European Heart Journal 200829 1224-1240. (doi:10.1093/ eurheartj/ehn156)

2 Pocock SJ, Wang D, Pfeffer MA, Yusuf S, McMurray JJ, Swedberg KB, Ostergren J, Michelson EL, Pieper KS \& Granger CB. Predictors of mortality and morbidity in patients with chronic heart failure. European Heart Journal 200627 65-75. (doi:10.1093/eurheartj/ehi555)

3 Shindler DM, Kostis JB, Yusuf S, Quinones MA, Pitt B, Stewart D, Pinkett T, Ghali JK \& Wilson AC. Diabetes mellitus, a predictor of morbidity and mortality in the Studies of Left Ventricular Dysfunction (SOLVD) Trials and Registry. American Journal of Cardiology 1996 77 1017-1020. (doi:10.1016/S0002-9149(97)89163-1)

4 Nichols GA, Gullion CM, Koro CE, Ephross SA \& Brown JB. The incidence of congestive heart failure in type 2 diabetes: an update. Diabetes Care 200427 1879-1884. (doi:10.2337/diacare.27.8. 1879)

5 Kannel WB, Hjortland M \& Castelli WP. Role of diabetes in congestive heart failure: the Framingham study. American Journal of Cardiology 197434 29-34. (doi:10.1016/0002-9149(74)90089-7)

6 McKee PA, Castelli WP, McNamara PM \& Kannel WB. The natural history of congestive heart failure: the Framingham study. New England Journal of Medicine 1971285 1441-1446. (doi:10.1056/ NEJM197112232852601)

7 Grundy SM, Benjamin IJ, Burke GL, Chait A, Eckel RH, Howard BV, Mitch W, Smith SC Jr \& Sowers JR. Diabetes and cardiovascular disease: a statement for healthcare professionals from the American Heart Association. Circulation 1999100 1134-1146. (doi:10.1161/01.CIR.100.10.1134)

8 Stone PH, Muller JE, Hartwell T, York BJ, Rutherford JD, Parker CB, Turi ZG, Strauss HW, Willerson JT, Robertson T, Braunwald E, Jaffe AS \& MILIS study group. The effect of diabetes mellitus on prognosis and serial left ventricular function after acute myocardial infarction: contribution of both coronary disease and diastolic left ventricular dysfunction to the adverse prognosis. The MILIS Study Group. Journal of the American College of Cardiology. 198914 49-57. (doi:10.1016/0735-1097(89)90053-3)

9 He J, Ogden LG, Bazzano LA, Vupputuri S, Loria C \& Whelton PK. Risk factors for congestive heart failure in US men and women: NHANES I epidemiologic follow-up study. Archives of Internal Medicine 2001161 996-1002. (doi:10.1001/archinte.161.7.996)

10 Rubler S, Dlugash J, Yuceoglu YZ, Kumral T, Branwood AW \& Grishman A. New type of cardiomyopathy associated with diabetic glomerulosclerosis. American Journal of Cardiology 1972 30 595-602. (doi:10.1016/0002-9149(72)90595-4)

11 Sharir T, Germano G, Kavanagh PB, Lai S, Cohen I, Lewin HC, Friedman JD, Zellweger MJ \& Berman DS. Incremental prognostic value of post-stress left ventricular ejection fraction and volume by gated myocardial perfusion single photon emission computed tomography. Circulation 1999100 1035-1042. (doi:10.1161/ 01.CIR.100.10.1035)

12 Berman DS, Kiat H, Friedman JD, Wang FP, van Train K, Matzer L, Maddahi J \& Germano G. Separate acquisition rest thallium201/stress technetium-99m sestamibi dual-isotope myocardial perfusion single-photon emission computed tomography: a clinical validation study. Journal of the American College of Cardiology 1993 22 1455-1464. (doi:10.1016/0735-1097(93)90557-H)

13 Zellweger MJ, Kaiser C, Brunner-La Rocca HP, Buser PT, Osswald S, Weiss P, Mueller-Brand J \& Pfisterer ME. Value and limitations of target-vessel ischemia in predicting late clinical events after drugeluting stent implantation. Journal of Nuclear Medicine $2008 \mathbf{4 9}$ 550-556. (doi:10.2967/jnumed.107.046771)

14 Gibbons RJ, Balady GJ, Bricker JT, Chaitman BR, Fletcher GF, Froelicher VF, Mark DB, McCallister BD, Mooss AN, O'Reilly MG, Winters WL Jr, Gibbons RJ, Antman EM, Alpert JS, Faxon DP, Fuster V, Gregoratos G, Hiratzka LF, Jacobs AK, Russell RO \& 
Smith SC Jr. ACC/AHA guideline update for exercise testing: summary article: a report of the American College of Cardiology/American Heart Association Task Force on Practice Guidelines (Committee to Update the 1997 Exercise Testing Guidelines). Circulation 2002106 1883-1892. (doi:10.1161/01.CIR. $0000034670.06526 .15)$

15 Zellweger MJ, Tabacek G, Zutter AW, Weinbacher M, Cron TA, Muller-Brand J \& Pfisterer ME. Evidence for left ventricular remodeling after percutaneous coronary intervention: effect of percutaneous coronary intervention on left ventricular ejection fraction and volumes. International Journal of Cardiology 200496 197-201. (doi:10.1016/j.ijcard.2003.06.016)

16 Berman DS, Hachamovitch R, Kiat H, Cohen I, Cabico JA, Wang FP, Friedman JD, Germano G, Van Train K \& Diamond GA. Incremental value of prognostic testing in patients with known or suspected ischemic heart disease: a basis for optimal utilization of exercise technetium-99m sestamibi myocardial perfusion single-photon emission computed tomography. Journal of the American College of Cardiology 199526 639-647. (doi:10.1016/0735-1097(95)00218-S)

17 Hachamovitch R, Berman DS, Kiat H, Cohen I, Cabico JA, Friedman J \& Diamond GA. Exercise myocardial perfusion SPECT in patients without known coronary artery disease: incremental prognostic value and use in risk stratification. Circulation 199693 905-914. (doi:10.1161/01.CIR.93.5.905)

18 Germano G, Kiat H, Kavanagh PB, Moriel M, Mazzanti M, Su HT, Van Train KF \& Berman DS. Automatic quantification of ejection fraction from gated myocardial perfusion SPECT. Journal of Nuclear Medicine $1995362138-2147$.

19 Sciagra R, Berti V, Genovese S \& Pupi A. Reliability of myocardial perfusion gated SPECT for the reproducible evaluation of resting left ventricular functional parameters in long-term follow-up. European Journal of Nuclear Medicine and Molecular Imaging 2010 37 1722-1729. (doi:10.1007/s00259-010-1444-y)

20 Hovland A, Staub UH, Bjornstad H, Prytz J, Sexton J, Stoylen A \& Vik-Mo H. Gated SPECT offers improved interobserver agreement compared with echocardiography. Clinical Nuclear Medicine 2010 35 927-930. (doi:10.1097/RLU.0b013e3181f9ddfb)

21 Klocke FJ, Baird MG, Lorell BH, Bateman TM, Messer JV, Berman DS, O'Gara PT, Carabello BA, Russell RO Jr, Cerqueira MD, St John Sutton MG, DeMaria AN, Udelson JE, Kennedy JW, Verani MS, Williams KA, Antman EM, Smith SC Jr, Alpert JS, Gregoratos G, Anderson JL, Hiratzka LF, Faxon DP, Hunt SA, Fuster V, Jacobs AK, Gibbons RJ \& Russell RO. ACC/AHA/ASNC guidelines for the clinical use of cardiac radionuclide imaging - executive summary: a report of the American College of Cardiology/American Heart Association Task Force on Practice Guidelines (ACC/AHA/ASNC Committee to Revise the 1995 Guidelines for the Clinical Use of Cardiac Radionuclide Imaging). Journal of the American College of Cardiology 200342 1318-1333. (doi:10.1016/j.jacc.2003.08.011)

22 Chareonthaitawee P, Sorajja P, Rajagopalan N, Miller TD, Hodge DO, Frye RL \& Gibbons RJ. Prevalence and prognosis of left ventricular systolic dysfunction in asymptomatic diabetic patients without known coronary artery disease referred for stress single-photon emission computed tomography and assessment of left ventricular function. American Heart Journal $2007 \mathbf{1 5 4}$ 567-574. (doi:10.1016/j.ahj.2007.04.042)

23 Htay T, Mehta D, Heo J \& Iskandrian AE. Left ventricular function in patients with type 2 diabetes mellitus. American Journal of Cardiology 200595 798-801. (doi:10.1016/j.amjcard.2004.11. 043)

24 Konduracka E, Gackowski A, Rostoff P, Galicka-Latala D, Frasik W \& Piwowarska W. Diabetes-specific cardiomyopathy in type 1 diabetes mellitus: no evidence for its occurrence in the era of intensive insulin therapy. European Heart Journal $2007 \mathbf{2 8}$ 2465-2471. (doi:10.1093/eurheartj/ehm361)

25 Mustonen JN, Uusitupa MI, Laakso M, Vanninen E, Lansimies E, Kuikka JT \& Pyorala K. Left ventricular systolic function in middleaged patients with diabetes mellitus. American Journal of Cardiology 199473 1202-1208. (doi:10.1016/0002-9149(94)90182-1)
26 Henry RM, Paulus WJ, Kamp O, Kostense PJ, Spijkerman AM, Dekker JM, Nijpels G, Heine RJ, Bouter LM \& Stehouwer CD. Deteriorating glucose tolerance status is associated with left ventricular dysfunction - the Hoorn Study. Netherlands Journal of Medicine 200866 110-117.

27 Loimaala A, Groundstroem K, Majahalme S, Nenonen A \& Vuori I. Impaired myocardial function in newly onset type 2 diabetes associates with arterial stiffness. European Journal of Echocardiography 20067 341-347. (doi:10.1016/j.euje.2005.07.001)

28 Ammann P, Brunner-La Rocca H, Fehr T, Munzer T, Sagmeister M, Angehrn W \& Rickli H. Coronary anatomy and left ventricular ejection fraction in patients with type 2 diabetes admitted for elective coronary angiography. Catheterization and Cardiovascular Interventions 200462 432-438. (doi:10.1002/ccd.20135)

29 McDonagh TA, Morrison CE, Lawrence A, Ford I, Tunstall-Pedoe H, McMurray JJ \& Dargie HJ. Symptomatic and asymptomatic left-ventricular systolic dysfunction in an urban population. Lancet 1997350 829-833. (doi:10.1016/S0140-6736(97)03033-X)

30 Silva JA, Escobar A, Collins TJ, Ramee SR \& White CJ. Unstable angina. A comparison of angioscopic findings between diabetic and nondiabetic patients. Circulation 199592 1731-1736. (doi:10.1161/01.CIR.92.7.1731)

31 Ingelsson E, Sundstrom J, Arnlov J, Zethelius B \& Lind L. Insulin resistance and risk of congestive heart failure. Journal of the American Medical Association 2005294 334-341. (doi:10.1001/ jama.294.3.334)

32 Smooke S, Horwich TB \& Fonarow GC. Insulin-treated diabetes is associated with a marked increase in mortality in patients with advanced heart failure. American Heart Journal 2005149 168-174. (doi:10.1016/j.ahj.2004.07.005)

33 Witteles RM \& Fowler MB. Insulin-resistant cardiomyopathy clinical evidence, mechanisms, and treatment options. Journal of the American College of Cardiology 200851 93-102. (doi:10.1016/ j.jacc.2007.10.021)

34 Hildebrandt P, Wachtell K, Dahlof B, Papademitriou V, Gerdts E, Giles T, Oikarinen L, Tuxen C, Olsen MH \& Devereux RB. Impairment of cardiac function in hypertensive patients with type 2 diabetes: a LIFE study. Diabetic Medicine 200522 1005-1011. (doi:10.1111/j.1464-5491.2005.01564.x)

35 Chung AK, Das SR, Leonard D, Peshock RM, Kazi F, Abdullah SM, Canham RM, Levine BD \& Drazner MH. Women have higher left ventricular ejection fractions than men independent of differences in left ventricular volume: The Dallas Heart Study. Circulation 2006113 1597-1604. (doi:10.1161/CIRCULATIONAHA.105. 574400)

36 Hachamovitch R, Rozanski A, Shaw LJ, Stone GW, Thomson LE, Friedman JD, Hayes SW, Cohen I, Germano G \& Berman DS. Impact of ischaemia and scar on the therapeutic benefit derived from myocardial revascularization vs. medical therapy among patients undergoing stress-rest myocardial perfusion scintigraphy. European Heart Journal 201132 1012-1024. (doi:10.1093/ eurheartj/ehq500)

37 Zhang L, Zhang S, Jiang H, Sun A, Zou Y \& Ge J. Effects of statin treatment on cardiac function in patients with chronic heart failure: a meta-analysis of randomized controlled trials. Clinical Cardiology 201134 117-123. (doi:10.1002/clc.20821)

38 Andersson C, Weeke P, Pecini R, Kjaergaard J, Hassager C, Kober L \& Torp-Pedersen C. Long-term impact of diabetes in patients hospitalized with ischemic and non-ischemic heart failure. Scandinavian Cardiovascular Journal 201044 37-44. (doi:10. 3109/14017430903312438)

39 De Groote P, Lamblin N, Mouquet F, Plichon D, McFadden E, Van Belle E \& Bauters C. Impact of diabetes mellitus on long-term survival in patients with congestive heart failure. European Heart Journal 200425 656-662. (doi:10.1016/j.ehj.2004.01.010)

Received 16 June 2011

Accepted 8 September 2011 